\title{
Further Study on the Short-timed Peroxidase Reaction of Blood Leucocytes; Prolongation of the Reaction in Avitaminosis B.
}

44th Rerort of the Peroxidase Reaction.

By

Tamotsu Sczuki.

(鍮木 保)

(From the Department of Pediatrics, Faculty of Medicine, Tohoku

Imperial University, Sendai. Divector, Prof. A. Sato.)

\section{Introduction.}

Different modifications of the original method of the copper peroxidase reaction devised by Prof. Sato and Sekiya ${ }^{1)}$ have been reported by a number of authors in- and outside our country. A rery short-timed peroxidase method was reported in 1915 from our laboratory by K. Suzuki, ${ }^{2}$, who found in collaboration with Arakawa") that in B-avitaminotic pigeons blood leucocytes would become weakly peroxidase-stained with a short-timed peroxidase method. Chiba ${ }^{4}$ of our laboratory made a special study on the relation of a very shorttimed peroxidase reaction and the state of $\mathrm{B}$-avitaminosis in human cases as well as in pigeons. His conclusions are to the effect that: 1 ) Blood leucocytes of pigeons, fed on B-avitaminotic food, will become

1) A. Sato and S. Sekiy a, Tohoku Igaku Zasshi, 1924, 6, 534 and Tohoku J. Exp. Med., 1926, 7, 111. (Cf. also A. Sato and Sh. Yoshimatsu, Amer. J. Diseas. Children, 1925, 29, 301.) Sato and Sekiya's Method is as follows: To a blood smear apply Solntion I $(0.5 \%$ Copper sulphate solution). Pour off most of it and apply Solution II (Saturated benzidin solution, to which add 2 drops of $3 \%$ hydrogen peroxid per $100 \mathrm{cc}$.) for two minutes. Then wash with water. The peroxidase granules of the leucocytes are stained a deep bluish green.

2) K. S nzuki, Jikwa Zasshi, 1925, 1416 and Tohoku J. Exp. Med., 1932, 19, 262. In fact before him $\mathrm{H}$. O ha ra tried in 1922 a short-timed modification of S a to-Sekiy a's oliginal method, but the time of application of Solution II was 20 seconds, thus rely short. (Nippon-no Ikai, 1922, 23, 690).

3) K. S uzuki and T. Arakawa, Tohoku J. Exp. Med., 1930, 16, 228.

4) M. Chiba, Tohoku J. Exp. Med., 1932, 19, 282 and 486. 
weakly stained with the short-timed peroxidase method, but become normally stained again on the administration of vitamin $B$, and 2) blood leucocytes of human cases that have something to do with Bavitaminosis will show a prolongation of the short-timed peroxidase reaction, but on the administration of vitamin $\mathrm{B}$, the prolongation will be made good. He used in human cases always "three seconds method" as short-timed peroxidase reaction, because he believed that human neutrophile leucocy tes became just perceptibly stained with the "three seconds method." Three seconds was the time during which Solution II of the Sato-Sekiya stain was to be applied on a blood film, while Solution I might be applied for any number of seconds.

\section{Alethod of Our oun Short-timed Peroridase Reation.}

It may seem unnecessary to write about the short-timed peroxidase reaction, becanse my method is almost the same as Chiba's, yet I describe it here for the reason which I shall give later.

The method used was as follows:

1. The direction of use of Solution I was entirely the same as in the original method. Stain time of Solution II was shortened to three or two seconds (timed by use of a chronometer), while with the original method it is as long as two minutes. Three or two seconds is the time when the neutrophile leucocytes on a fresh blood smear from a healthy individual begin to take the stain.

2. Solution II was prepared fresh at each examination. This was again different from the original method, in which there is no absolute necessity for a fresh preparation.

3. Blood smears were tested within a definite time; always within two hours of blood taking.

4. The reaction was tested always at the room temperature.

A large number of blood smear's were prepared from each patient, so that a good smear, homogenous and not too thick, might be chosen from among them. Neutrophiles were made the objects of microscopic examination.

The original copper method of Sato and Sekiya, i.e. with $2 \mathrm{~min}$ utes stain time of Solution II, stains myeloid leucocytes distinctly greenish-blue so that in general no one will have them stained any longer. But this uriginal method stains blood leucocytes in both normal and B-avitaminotic subjects were equally distinctly greenish-blue. The difference between normal and B-avitaminotic leucocytes will, as 
stated above, be shown only with a very short-timed peroxidase reaction. And Chiba used the "three seconds method" exclusively, because he considered 3 seconds as the shortest possible time for copper peroxidase reaction (with the Solution II).

I learned* the "three seconds method" first, but after some months of practice, I have come to consider 2 seconds for Solution II as the normal shortest stain time.

In the former series (Part I) of my own cases the normal shortest stain time for peroxidase reaction was three seconds, and in the latter series (Part II) two seconds. In my future reports concerning the shortest peroxidase-stain time, this of course will always remain two seconds instead of three.

\section{PART I.}

Three Seconds as the Shortest Stain Time for Copper

Peroxidase Reaction.

In Part I the shortest time, viz. the normal time for the peroxidase reaction was considered to be three seconds.

\section{Cases of Sucklings with Apparent B-deficit and with Nutritional Disturbance.}

These cases were fed with an apparently false formula with an evident B-deficit, and there were six cases $(40 \%)$ with a prolongation of the shortest peroxidase stain time. And two cases (Cases Nos. 6 $\& 8$ in Table I) with such a prolongation showed, when tested later after some time of a daily administration of vitamin B, no longer any prolongation. I was unable to test the other cases. Cf. Table I.

\section{Cases of Allaitement Mixte (with Such Human Milk either Negative or Weakly Positive to A rakawa's Reactions).}

In these 16 cases with allaitement mixte there were 9 cases $(56.2 \%)$ with a prolongation, of which 3 cases were fed on human milk entirely or almost negative to Arakawa's reaction. And I was able to test

* The original method of Sato and Sekiy a is very easy and can be performed with success by a beginner, while such a very short-timed method as the three seconds one can be attained only after long practice.

$\$$ T. Arakawa, Tohoku J. Exp. Med., 1930, 16, 83 and 107. 


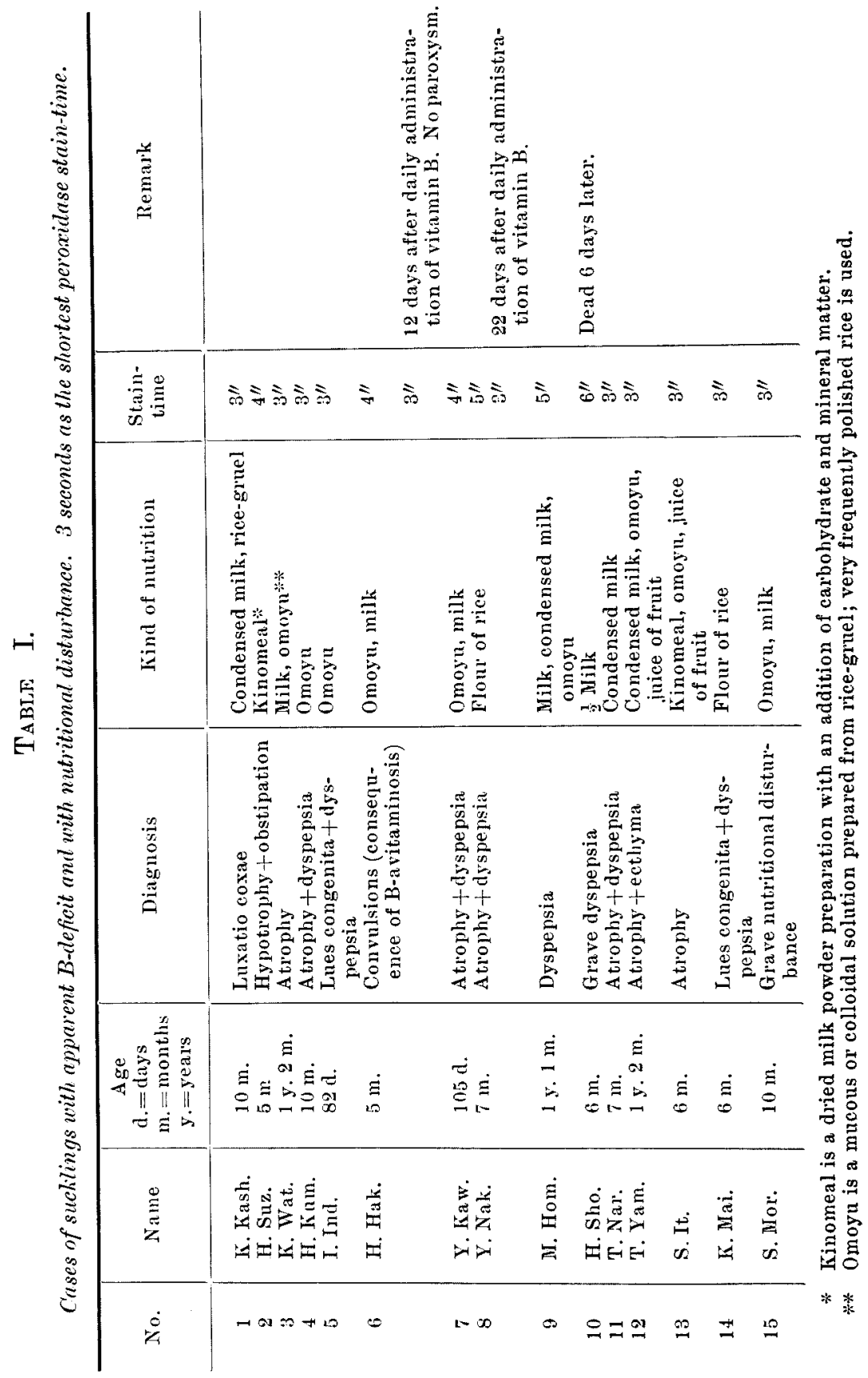




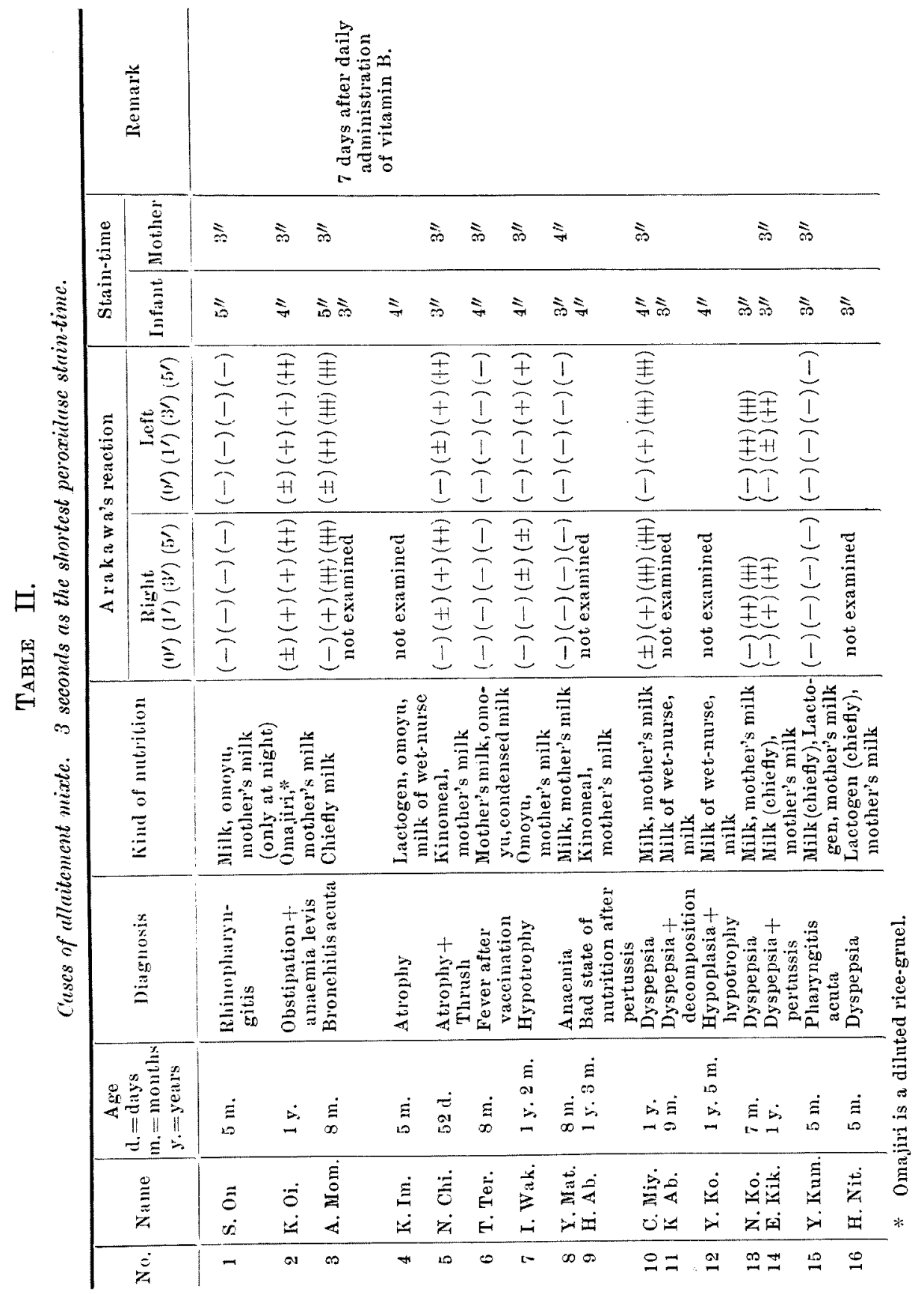


only one case (Case No. 3 in Table II) again after a week of daily administration of vitamin $B$, when it no longer showed any prolongation of the shortest peroxidase stain time. And it is to be noted here that most of the infants in Table II suffered from nutritional disturbance. Cf. Table II.

\section{Cases of Breast-fed Infants with Human Milk (either.}

Negative or ITeakly Positive to Arakawa's Reaction).

In these 24 cases of breast feeding, there were 6 infants $(25 \%)$ with a prolongation of the shortest peroxidase stain time. And 18 out of these 24 suffered from nutritional disturbance of some kind or other. It was possible to test Cases No. 1 and No. 3 in Table III again after resp. 4 and 33 days of daily administration of vitamin $B$, when their shortest peroxidase stain time was already restored to 3 seconds (thus to normal). Cf. Table III.

$$
\text { PART II. }
$$

Two Seconds as the Shortest Stain Time for Copper

Peroxidase Reaction.

While $I$ was working on the shortest stain time for Sato and Sekiya's copper peroxidase reaction, I have, as stated before, come to consider not three seconds, but two seconds as the shortest possible stain-time for it, because neutrophile leucocytes are just perceptibly blue-stained during two seconds' time. And I have put the new method to test in similar cases as those shown in Part I.

\section{Cases of Sucklings with Apparent B-deficit and with Nutritional Disturbance.}

There were four cases $(30.8 \%)$ with a prolongation of the shortest peroxidase stain-time. And one case (Case No. 10 in Table IV), which was the only case I was able to examine more than once, showed, as will be seen from Table IV, a gradual restoration of the prolonged peroxidase stain-time to the normal. This case will again show that an administration of vitamin $B$ in a large amount will bring back a prolonged peroxidase stain-time to the normal. Cf. Table IV. 


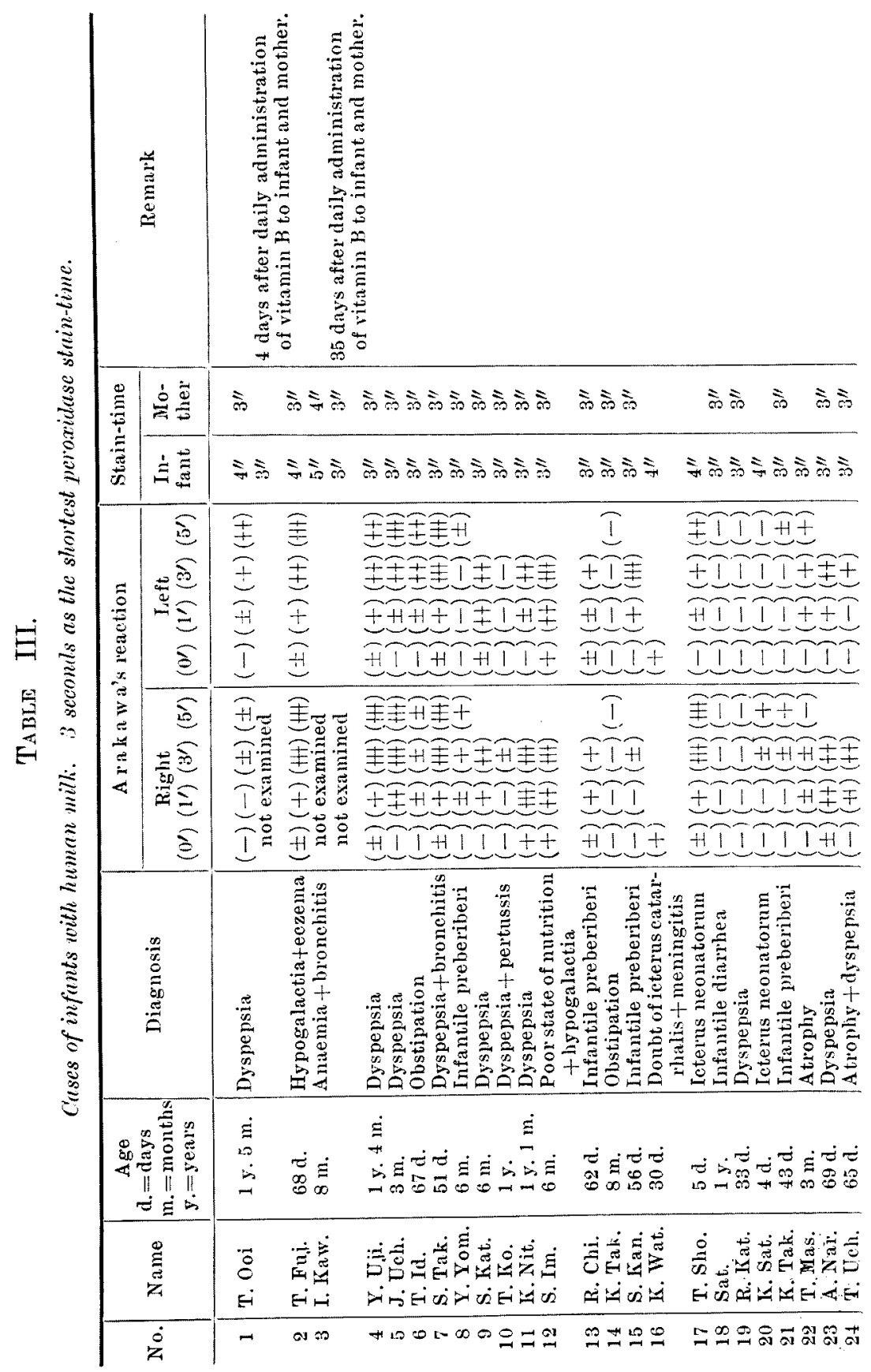




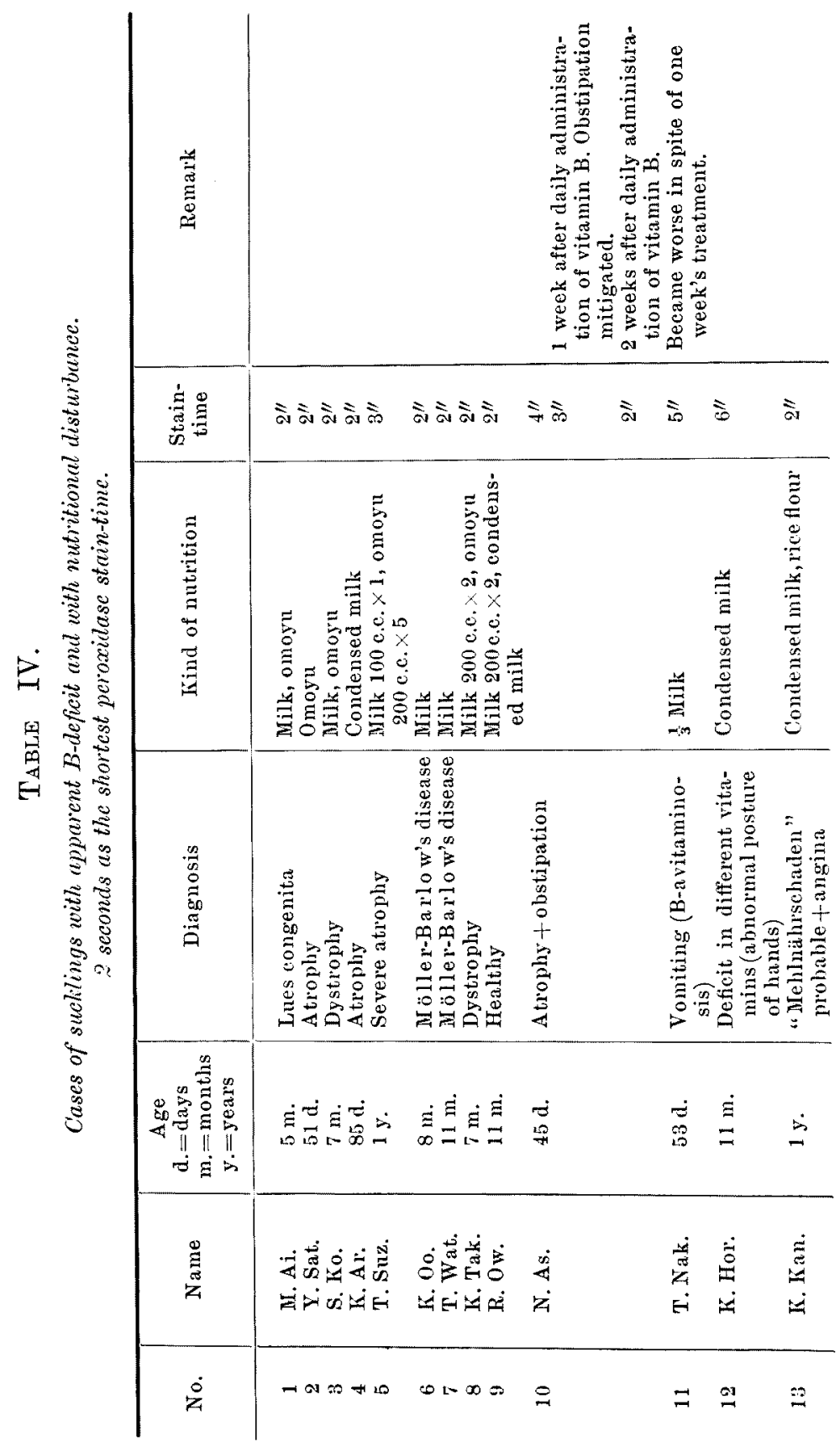




\section{Cases of Allaitement Mixte (with such a Human Milk as was either Negative or Weakly Positive to Arakawa's Reaction).}

Most of the cases tabulated in Table V suffered from nutritional disturbance, and there was 9 cases (39.1\%) with a prolongation of the shortest peroxidase stain-time, of which 5 cases were fed on human milk with completely or almost negative to Arakawa's reaction. And I was able to test 2 cases (Cases No. 2 and No. 10 in Table V) again after some days of daily administration of vitamin $B$ to mothers and infants, when a prolongation was no longer seen in either of them. Cf. Table V.

III. Cases of Breast-fed Infants with Human Milk (either Negative or Weak to Arakaw a's Reaction).

Of these 81 cases with exclusive breast feeding there were 72 cases with negative or weakly positive to A rakawa's reaction, and 37 cases $(51.4 \%)^{*}$ had a prolongation of the shortest peroxidase staintime and most of these suffered from nutritional disturbance of some kind or other, especially from infantile beriberi or infantile preberiberi. Of these 37 cases I was able to examine the shortest stain-time in 8 cases after a lapse of time. All the cases except two were restored to the normal as to the peroxidase reaction after from 4 to 11 days of daily administration of vitamin $B$, and in one case (Case No. 16 in Table VI) of the two exceptions the shortest stain-time $\left(4^{\prime \prime}\right)$ was shortened to three seconds, so that it may be assumed that it must have been restored to normal (two seconds' time) later after a continuous use of the vitamin B. And in the other case (Case No. $47 \mathrm{in}$ Table VI) the shortest peroxidase stain-time $\left(3^{\prime \prime}\right)$ was gradually prolonged up to five seconds and the infant began to vomit in spite of daily administration of vitamin $B$ to mother and infant, probably because the mother suffered at that time more severely from the disease, which presumption was not without reason from the fact that the prolongation of the shortest peroxidase stain-time had now begun to manifest itself in the mother's blood too. More interesting is the case No. 35 in Table VI, in which the stain-time of the infant's blood was two seconds at the time when infant and mother were taking vitamin $B$, while, after giving up vitamin $B$ for a week, the stain-time

* In contrast to $37.0 \%$ of $\mathrm{Ch}$ iba ; Cf. M. Chi ba, Tohoku J. Exp. Med., 1932, 19, 487. 


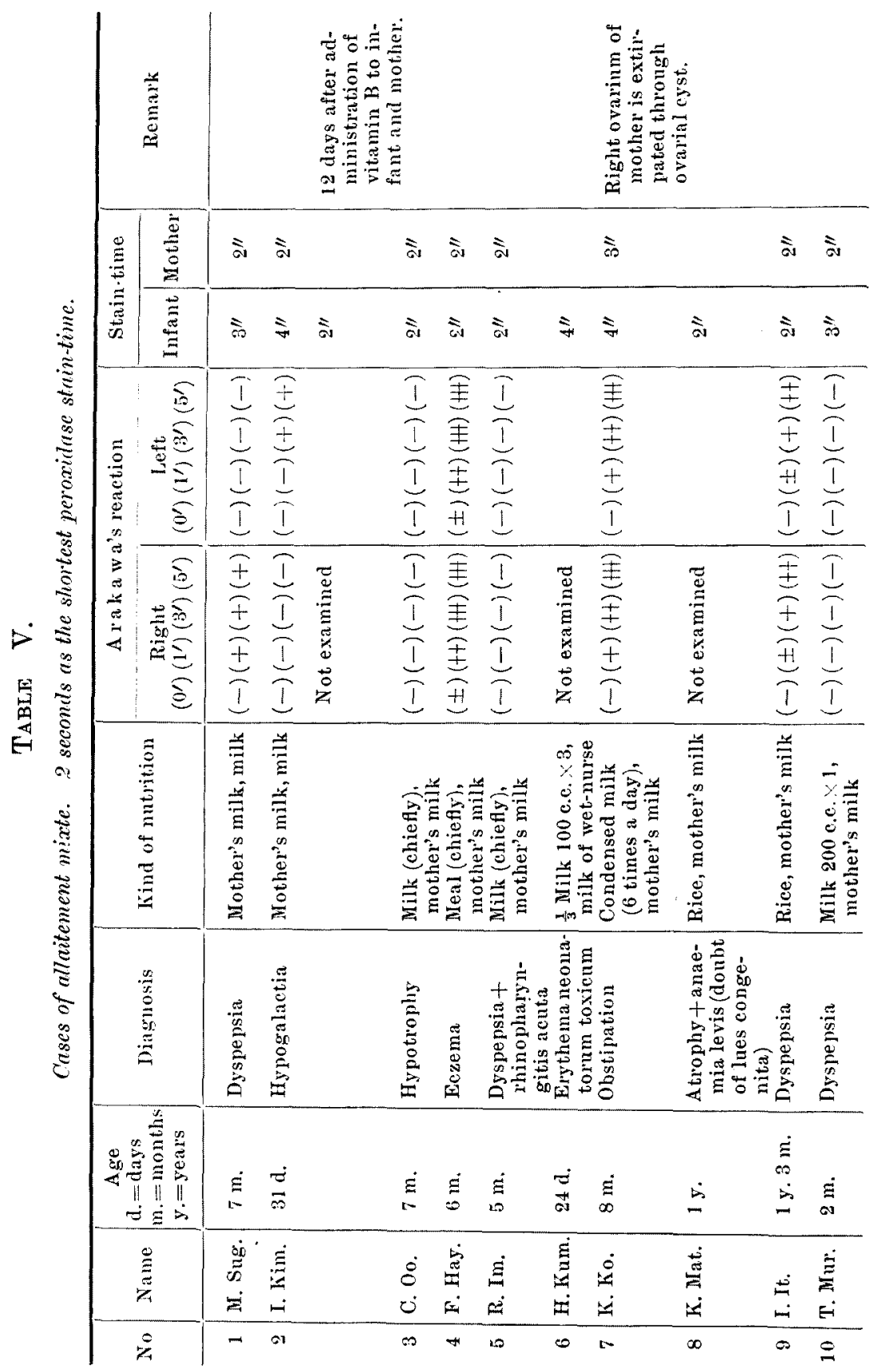



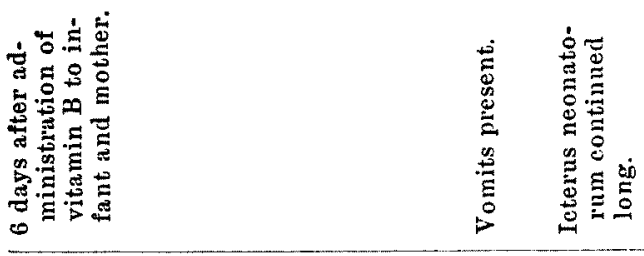
สे जे जे जे ิे
ลे
ธे
जे

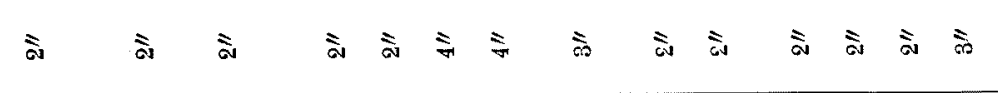

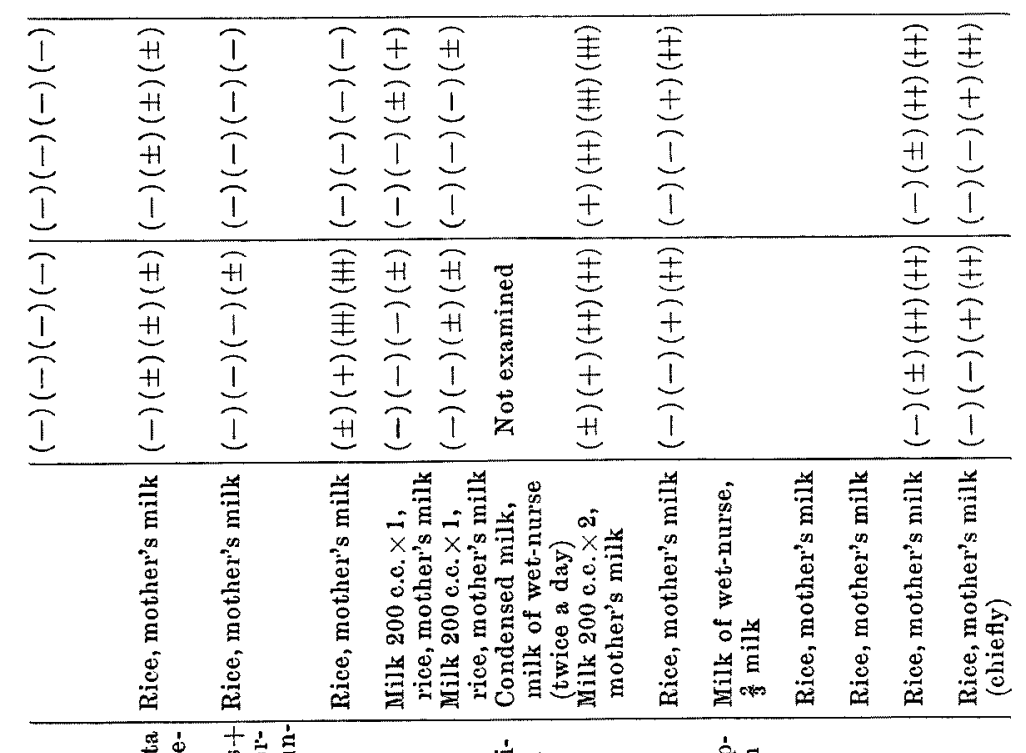

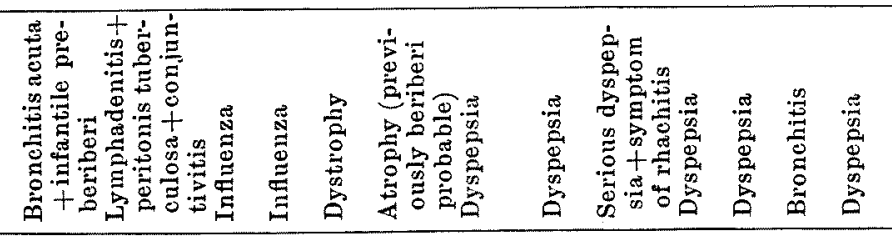

\begin{tabular}{|c|c|c|c|c|c|c|c|c|c|c|c|c|}
\hline $\begin{array}{l}\dot{\Xi} \\
m \\
\dot{\infty} \\
\sim\end{array}$ & $\begin{array}{l}\dot{g} \\
\text { a } \\
\dot{\sim} \\
\rightarrow\end{array}$ & $\begin{array}{l}\dot{g} \\
\sim \\
\dot{ } \\
\sim\end{array}$ & 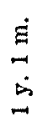 & $\begin{array}{l}\text { ri } \\
\text { Nֶ }\end{array}$ & ম્ય & 客 & $\dot{\vec{\Delta}}$ & 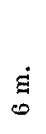 & $\begin{array}{l}\dot{g} \\
\dot{\vec{g}} \\
\dot{\sigma}\end{array}$ & 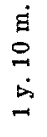 & $\begin{array}{l}\underset{j}{\dot{j}} \\
\underset{\mathbf{D}}{\Delta}\end{array}$ & $\begin{array}{l}\dot{g} \\
\dot{0} \\
\dot{ }\end{array}$ \\
\hline 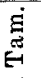 & 苞 & ஜूं & 离 & 我 & $\dot{\Xi}$ & $\dot{\vec{\theta}}$ & 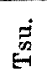 & 离 & 菏 & 苛 & $\dot{\ddot{\theta}}$ & $\begin{array}{l}\dot{H} \\
\text { Ë }\end{array}$ \\
\hline$\dot{H}$ & $\dot{\infty}$ & $\dot{\nexists}$ & 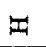 & $\ddot{\mid r}$ & $\dot{4}$ & $i$ & 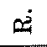 & $\ddot{\Delta}$ & 它 & $\dot{4}$ & $E-$ & 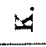 \\
\hline$\Rightarrow$ & $\underset{\sim}{\stackrel{N}{1}}$ & $\cong$ & $\Xi$ & 20 & $\mathscr{0}$ & $\Xi$ & $\stackrel{\infty}{=}$ & $\stackrel{\theta}{\sigma}$ & \& & $\vec{a}$ & gี & $\approx$ \\
\hline
\end{tabular}




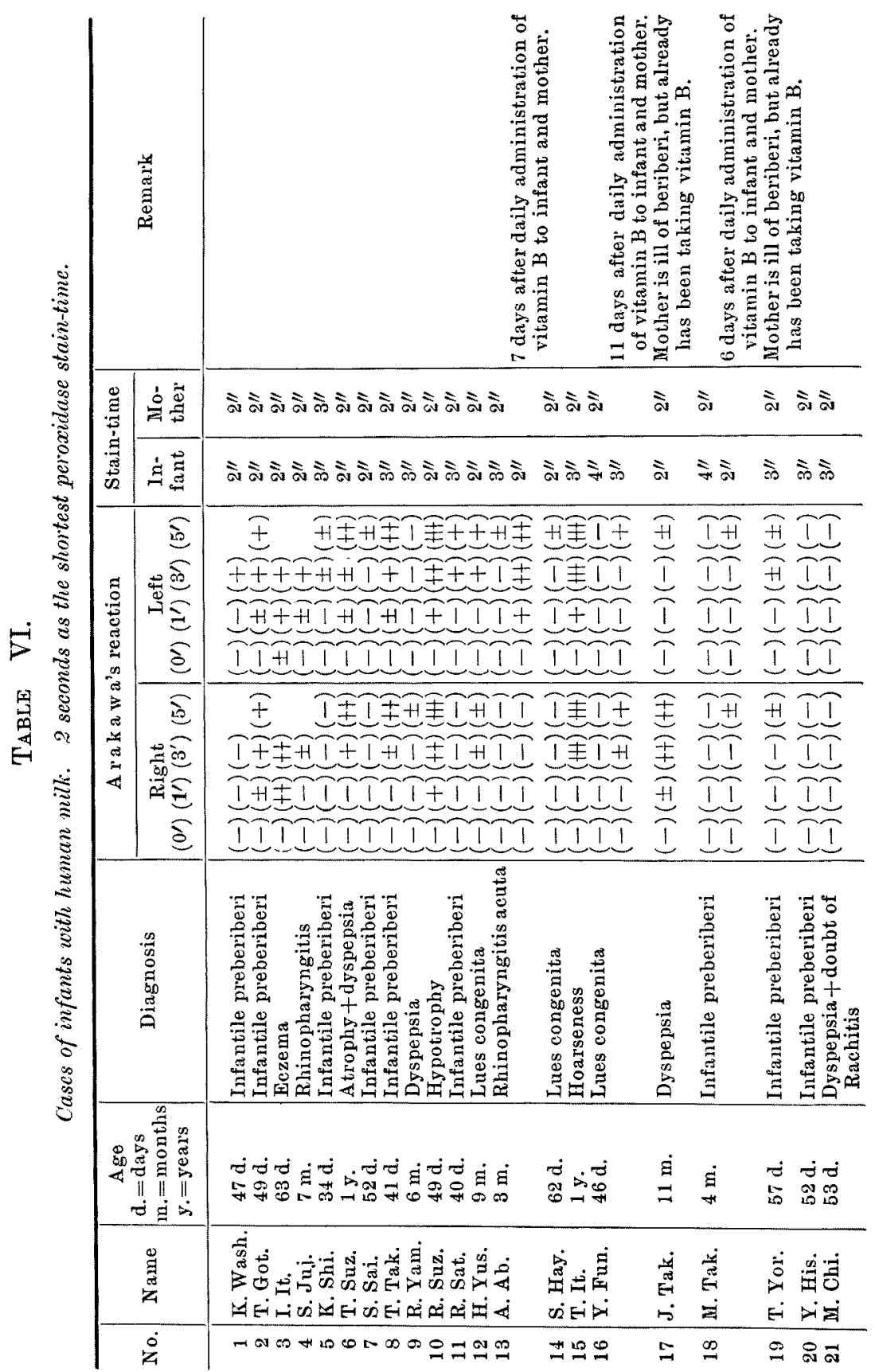




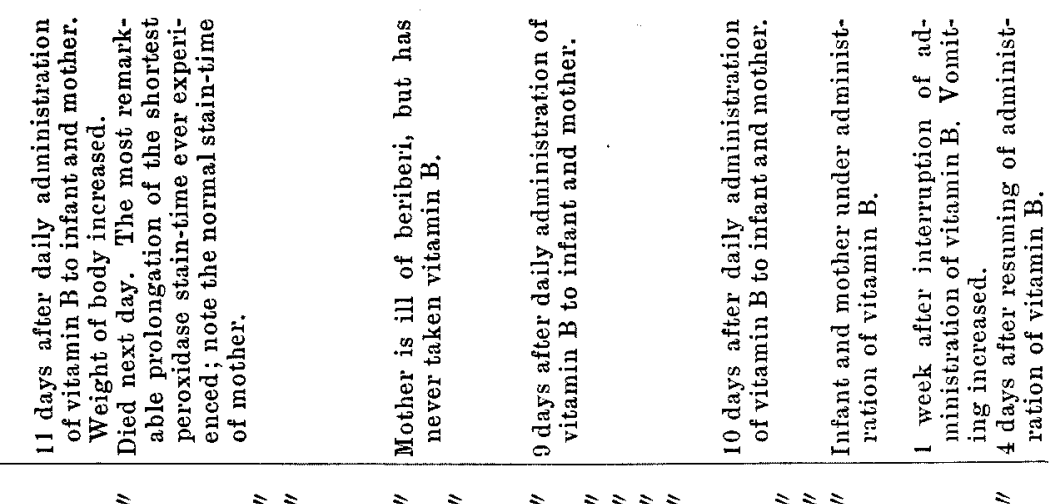

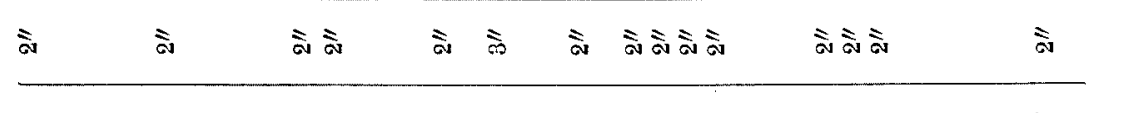

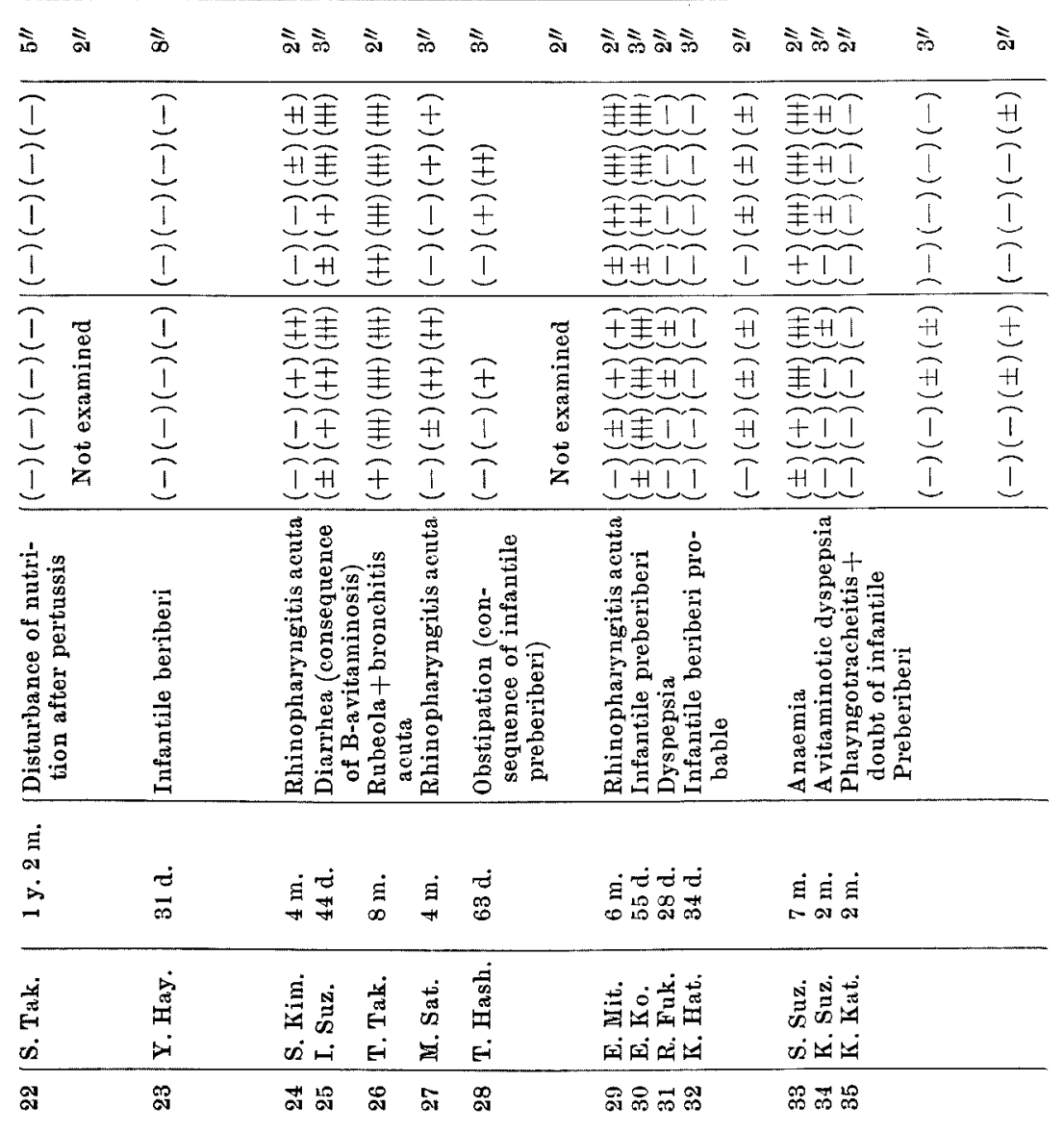




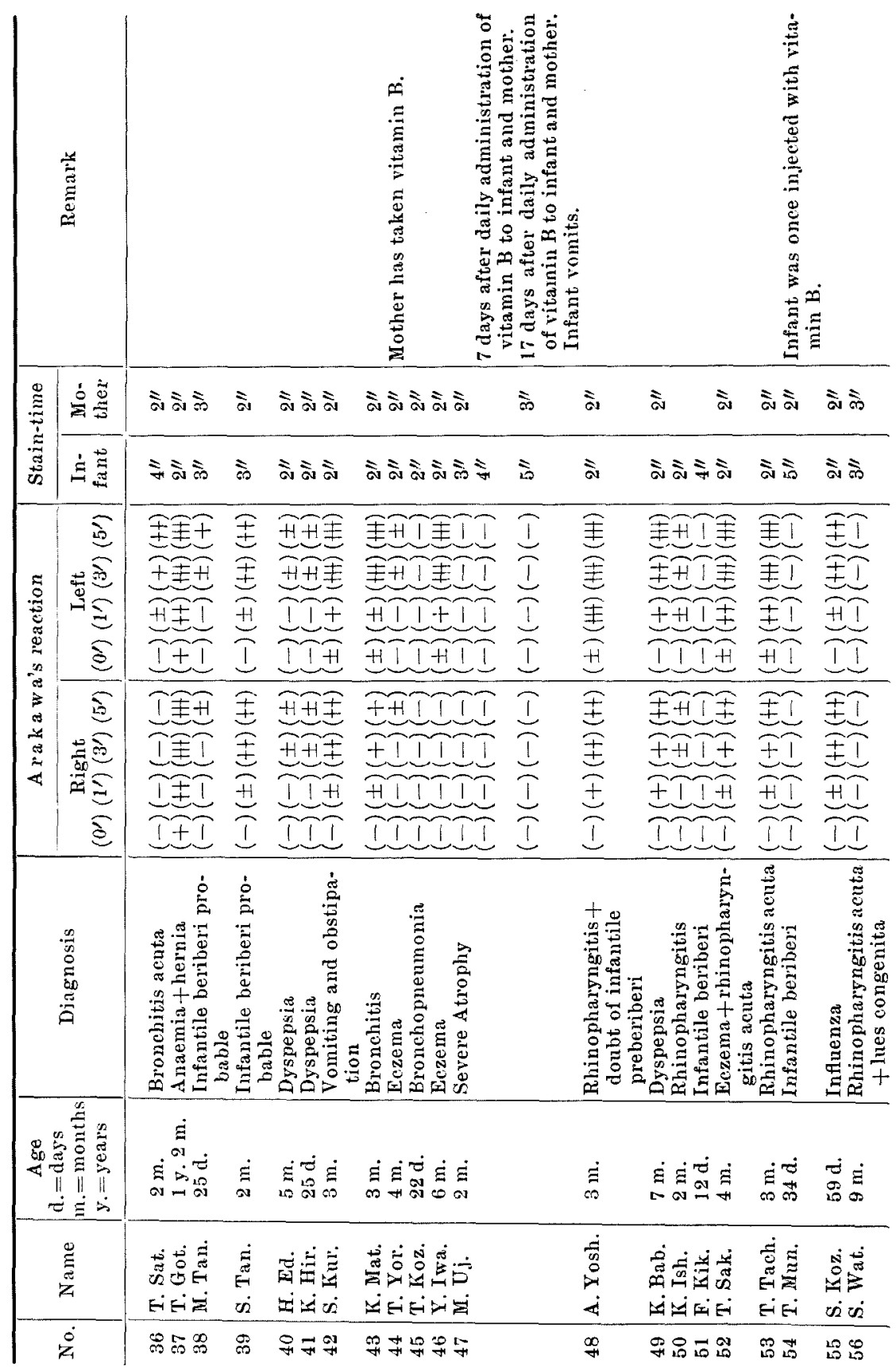




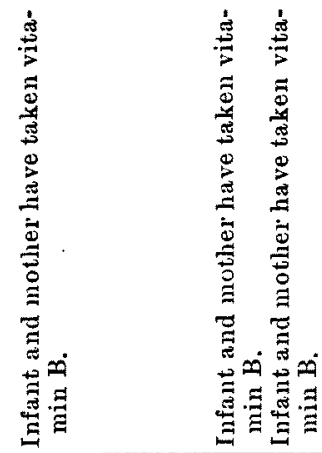

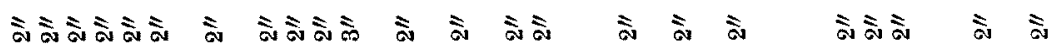

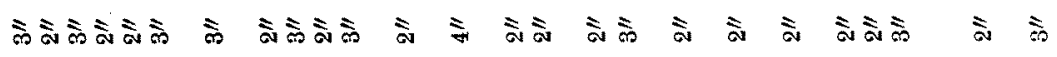

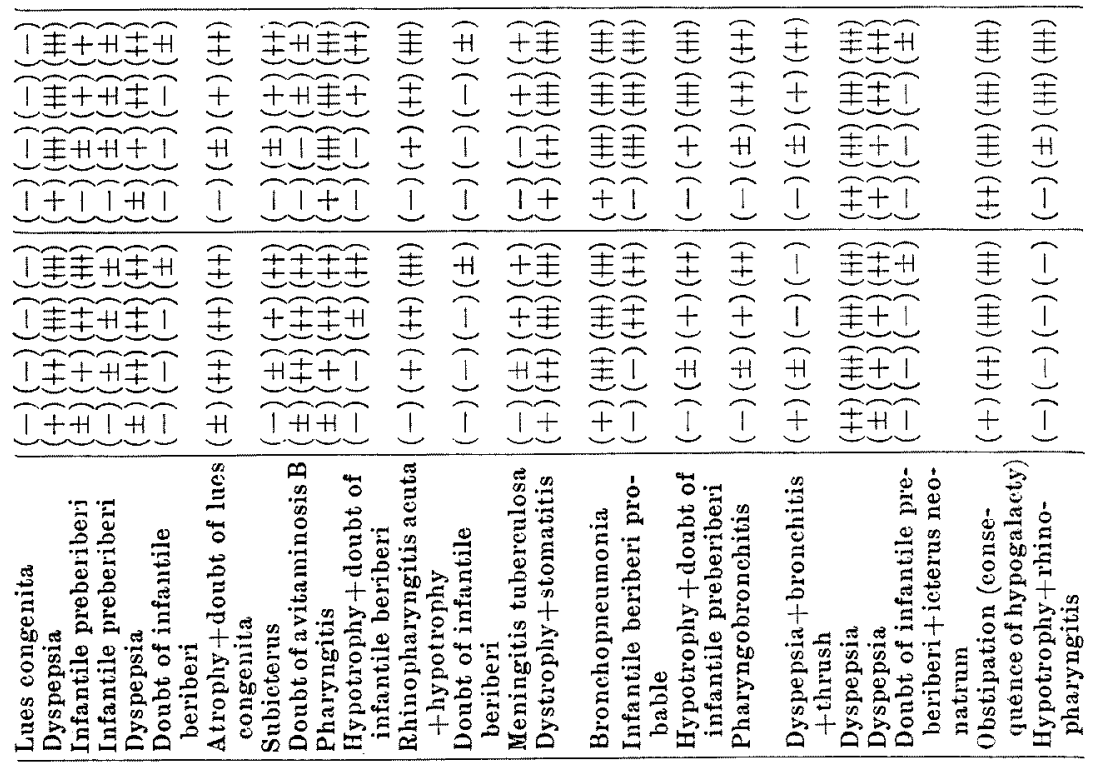

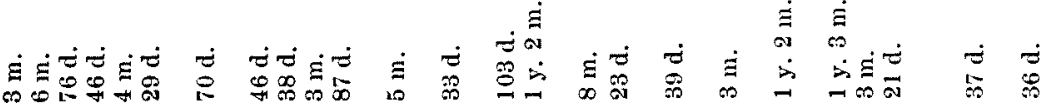

\begin{tabular}{|c|c|c|c|c|c|c|c|c|c|c|c|}
\hline 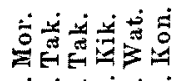 & 車 & 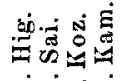 & 总 & 离 & 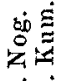 & $\dot{\Xi}$ & $\because$ & 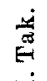 & 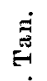 & $\dot{0}$ & $\ddot{\theta}$ \\
\hline 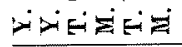 & $\therefore$ & 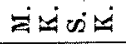 & $E$ & $\dot{1}$ & $\infty 0^{\circ}$ & $E \dot{x}$ & $\dot{x i}$ & $\dot{z}$ & $\dot{n}$ & EAERE & $\dot{z}$ \\
\hline & & त् & & & & & & & & & \\
\hline
\end{tabular}


$\left(2^{\prime \prime}\right)$ was prolonged to three seconds, and then, after taking vitamin $\mathrm{B}$ again for 4 days the prolonged stain-time $\left(3^{\prime \prime}\right)$ was shortened again and restored to the normal.

It is to be noted here that mothers were, from the peroxidase point of view, suffering from avitaminosis $B$ in a less degree than their babies were, because there were only 6 cases $(9.1 \%)^{*}$ with a prolongation of the shortest peroxidase stain-time out of 65 mothers, who were secreting milk negative or weakly positive to Arakawa's reaction. And we can say with $\mathrm{Chiba}$ and $\mathrm{Ab} \mathrm{e}^{5)}$ that " assuming that the prolongation of the stain-time is due to the grade of avitaminosis $\mathrm{B}$, it is a possibility that a mother secreting B-avitaminotic milk can make her suckling more avitaminotic than herself." I was able to examine 17 babies of infantile preberiberi and among them $I$ found 11 cases with a prolongation of the shortest peroxidase stain-time. $\left(\mathrm{Chiba}^{4)}\right.$ examined 8 cases of infantile preberiberi, all with a prolongation of the shortest peroxidase stain-time).

As to the case of the diagnosis of infantile beriberi and that of infantile B-avitaminosis, there was no case out of all the 13 patients without a prolongation of the shortest peroxidase stain-time; the stain-time was 3 seconds in nine cases, 4 seconds in two, 5 seconds in one, and in the other remaining case, as long as 8 seconds. But then, there were only two out of the whole 13 mothers with a prolongation of the shortest peroxidase stain-time. $\mathrm{Chiba}^{4)}$ examined 17 cases of infantile beriberi and there were 9 babies and only 2 mothers with a prolongation of the shortest peroxidase stain-time. Here again I repeat the above cited quotation from the paper of Chiba and Abe.5) As to the shortest possible peroxidase stain-time as my experience described in Part II shows, it is two seconds instead of three, and two seconds will be considered as normal for the short-timed peroxidase stain.

\section{Final Comparison between Two and Three Sceonds Method in Different Diseases.}

Here a comparison between the "two" and the "three seconds method" in different diseases will be given. This comparison is not, however, that between the two methods in one and the same cases, but that between the "three seconds method" in one series of cases

* In contrast to $17.0 \%$ of Chiba; Cf. M. Chiba, Tohoku J. Exp. Med., 1932, 19, 492.

5) M. Chib a and J. A be, Tohoku J. Exp. Med., 1932, 19, 479. 
TABLE VII.

Cases of different diseases including healthy. 3 seconds as the shortest peroxidase stain-time.

\begin{tabular}{|c|c|c|c|c|c|}
\hline No. & Name & \begin{tabular}{|}
$\quad$ Age \\
d.=days \\
m.=months \\
y.= =years
\end{tabular} & Diagnosis & $\begin{array}{l}\text { Stain- } \\
\text { time }\end{array}$ & Remark \\
\hline 1 & K. Mor. & $4 \mathrm{~d}$. & $\begin{array}{l}\text { Melaena neonatorum }+ \\
\text { ieterus neonatorum }\end{array}$ & $3^{\prime \prime}$ & \\
\hline 2 & T. Sho. & $1 \mathrm{y} .5 \mathrm{~m}$. & Dyspepsia & $3^{\prime \prime}$ & \\
\hline 3 & T. Sat. & 1 y. $7 \mathrm{~m}$. & , & $3 \prime \prime$ & \\
\hline 4 & Y. Kat. & $1 \mathrm{y} .6 \mathrm{~m}$. & $"$ & $3^{\prime \prime}$ & \\
\hline 5 & T. Mut. & $1 \mathrm{y} .6 \mathrm{~m}$. & Dyspepsia-thrush & $3^{\prime \prime}$ & \\
\hline 6 & T. Um. & $9 \mathrm{y} .3 \mathrm{~m}$. & Beriberi & $3^{\prime \prime}$ & $\begin{array}{l}\text { Has taken vitamin B } \\
\text { and "Haiga-mai." }\end{array}$ \\
\hline 7 & T. Wat. & $25 \mathrm{y}$. & $"$ & $3^{\prime \prime}$ & $\begin{array}{l}\text { Has been taking vita- } \\
\text { min } B \text {. }\end{array}$ \\
\hline 8 & I. Tak. & $11 \mathrm{~g} .7 \mathrm{~m}$. & Gastritis acuta & $3^{\prime \prime}$ & \\
\hline 9 & M. Jo. & $3 y$. & $"$ & $3^{\prime \prime}$ & \\
\hline 10 & Y. Nun. & $7 \mathrm{y} .7 \mathrm{~m}$. & "ctelns "arre & $3^{\prime \prime}$ & \\
\hline & & & icherus caldarinalis & $3^{\prime \prime}$ & $\begin{array}{l}12 \text { days later. jaundice } \\
\text { disappeared. }\end{array}$ \\
\hline 12 & I. Sat. & $6 \mathrm{~s} .9 \mathrm{~m}$. & $"$ & $3^{\prime \prime}$ & \\
\hline 13 & J. Uch. & $50 \mathrm{~d}$. & $"$ & $3^{\prime \prime}$ & $\begin{array}{l}\text { Jaundice almost disap- } \\
\text { peared. }\end{array}$ \\
\hline 14 & I. Hat. & $5 \mathrm{y} .5 \mathrm{~m}$. & Angina acuta & $3^{\prime \prime}$ & \\
\hline 15 & C. $A b$. & $5 \mathrm{y} .5 \mathrm{~m}$ & Rhinopharyngitis acuta & $3^{\prime \prime}$ & \\
\hline 16 & K. Ab. & $2 \mathrm{y} .10 \mathrm{~m}$. & Pharyngitis acuta & $3^{\prime \prime}$ & \\
\hline 17 & H. Fuk. & $1 \mathrm{y.} 4 \mathrm{~ms}$. & Rhinopharyngitis acuta & $3^{\prime \prime}$ & \\
\hline 18 & I. Tak. & $9 \mathrm{y}$. & Hypertrophy of tonsil & $3^{\prime \prime}$ & \\
\hline 19 & N. I wa. & $5 \mathrm{y} .8 \mathrm{~m}$. & Bronchitis acuta & $3^{\prime \prime}$ & \\
\hline 20 & S. Ab. & $8 \mathrm{y}, 2 \mathrm{~m}$. & Periodic vomiting & $\begin{array}{l}4^{\prime \prime} \\
3^{\prime \prime}\end{array}$ & $\begin{array}{l}9 \text { days after administra- } \\
\text { tion of vitamin } B \text { and } \\
\text { injection of Yakriton } \\
\frac{1}{3} \text { unit once. }\end{array}$ \\
\hline 21 & T. Tak. & $9 \mathrm{y} .10 \mathrm{~m}$. & Orthotic albuminuria & $3^{\prime \prime}$ & \\
\hline 22 & T. Ito. & $4 \mathrm{~m}$ & Meningitis purulenta & $3^{\prime \prime}$ & \\
\hline 23 & H. Ot. & $8 \mathrm{y} .6 \mathrm{~m}$ & $\begin{array}{l}\text { Choreic movement } \\
\text { weakness of mind (con- } \\
\text { sequence of encephalitis) }\end{array}$ & $3^{\prime \prime}$ & $\begin{array}{l}\text { Administration of vita- } \\
\text { min } B \text { and injection of } \\
\text { Yakriton } 1 \text { unit twice. } \\
9 \text { days after choreic } \\
\text { movement decreased. }\end{array}$ \\
\hline 24 & M. Mor. & $14 \mathrm{y}$ & Chorea & $3^{\prime \prime}$ & \\
\hline 25 & J. Sai. & 2 y. $11 \mathrm{~m}$. & Dyspepsia acuta & $3^{\prime \prime}$ & \\
\hline 26 & E. Ko. & $5 \mathrm{y} .11 \mathrm{~m}$. &, & $3^{\prime \prime}$ & \\
\hline 27 & A. Tak. & 3 y. $11 \mathrm{~m}$. & $"$ & $3^{\prime \prime}$ & \\
\hline 28 & A. Ak. & $3 \mathrm{y} .5 \mathrm{~m}$ & $"$ & $3^{\prime \prime}$ & \\
\hline 29 & K. Rau. & $4 \mathrm{y} .10 \mathrm{~m}$ & $"$ & $3^{\prime \prime}$ & \\
\hline 30 & N. Nag. & $4 \mathrm{~F} .1 \mathrm{~m}$ & " & $3^{\prime \prime}$ & \\
\hline 31 & R. Asa. & $1 \mathrm{y} .11 \mathrm{~m}$. & " & $3^{\prime \prime}$ & \\
\hline 32 & C. Kum. & $9 \mathrm{s.1 \textrm {m }}$. & Latent tuberculosis & $3^{\prime \prime}$ & \\
\hline 33 & Y. Nar. & $1 \mathrm{y} .8 \mathrm{~m}$ & Erythema nodosum & $3^{\prime \prime}$ & \\
\hline 34 & H. Shib. & $1 \mathrm{~g} .10 \mathrm{~m}$. & Lues congenita & $3^{\prime \prime}$ & \\
\hline 35 & T. Tak. & $2 \mathrm{y} .11 \mathrm{~m}$. & Eczema & $3^{\prime \prime}$ & \\
\hline 36 & K. Wat. & $3 \mathrm{y} .3 \mathrm{~m}$ & Healtby & $3^{\prime \prime}$ & \\
\hline 37 & I. Tak. & $7 \mathrm{y} .3 \mathrm{~m}$. & , & $3^{\prime \prime}$ & \\
\hline
\end{tabular}




\section{TABLE VIII.}

Cases of different diseases including healthy. 2 seconds as the shortest peroxidase stain-time.

\begin{tabular}{|c|c|c|c|c|c|}
\hline No. & Name & $\begin{array}{c}\text { Age } \\
\mathrm{d} .=\text { days } \\
\mathrm{m}=\text { months } \\
\mathrm{y} .=\text { years }\end{array}$ & Diagnosis & Stain- & Remark \\
\hline 1 & K. Nak. & $1 \mathrm{~g} .4 \mathrm{~m}$. & Möller-Barlow's disease & $2^{\prime \prime}$ & \\
\hline 2 & F. Suz. & $6 \mathrm{~m}$. & Lead-poisoning & $2 / \prime$ & \\
\hline 3 & K. Tak. & $9 \mathrm{~m}$ & Poisoning of human milk & $2^{\prime \prime}$ & \\
\hline 4 & K. Tsu. & $1 \mathrm{y} .5 \mathrm{~m}$. & Dyspepsia acuta & $2^{\prime \prime}$ & \\
\hline 5 & Y. Sun. & $5 \mathrm{y} .11 \mathrm{~m}$. & " $"$ & $2^{\prime \prime}$ & \\
\hline 6 & M. Tak. & $1 \mathrm{y} .3 \mathrm{~m}$ & $"$ & $2^{\prime \prime}$ & \\
\hline 7 & H. Ao. & $1 \mathrm{y} .4 \mathrm{~m}$. &, & $2^{\prime \prime}$ & \\
\hline 8 & $\mathbf{T} . \mathbf{A k}$ & 1 y. $8 \mathrm{~m}$. & Hypotrophy & $2^{\prime \prime}$ & \\
\hline 9 & K. Suz. & $13 \mathrm{y}$. & Appendicitis acuta & $2^{\prime \prime}$ & \\
\hline 10 & T. Suz. & $8 \mathrm{y} .11 \mathrm{~m}$. & Stomatitis & $2^{\prime \prime}$ & \\
\hline 11 & H. Hash. & $5 \mathrm{y} .4 \mathrm{~m}$ & Ascariasis & $2^{\prime \prime}$ & \\
\hline 12 & E. Ak. & $2 \mathrm{y} .10 \mathrm{~m}$. & $\begin{array}{l}\text { Obstinate chronic } \\
\text { diarrhea }\end{array}$ & $2^{\prime \prime}$ & \\
\hline 13 & M. Nak. & $3 \mathrm{y} .6 \mathrm{~m}$. & Abscess in abdomen & $2^{\prime \prime}$ & \\
\hline 14 & H. U.j. & 10 y. $2 \mathrm{~m}$ & Icterus eatarrhalis & $2^{\prime \prime}$ & $\begin{array}{l}\text { Has been taking vita- } \\
\text { min } B \text {. }\end{array}$ \\
\hline 15 & S. Ko. & $4 \mathrm{y} .6 \mathrm{~m}$. & False croup & $2^{\prime \prime}$ & \\
\hline 16 & A. Mo. & $8 \mathrm{y} .2 \mathrm{~m}$. & Angina & $2^{\prime \prime}$ & \\
\hline 17 & A. Min. & $1 \mathrm{y} .2 \mathrm{~m}$. & ", & $2^{\prime \prime}$ & \\
\hline 18 & M. Sat. & $8 \mathrm{y} .1 \mathrm{~m}$. & Bronchitis acuta & $2^{\prime \prime}$ & \\
\hline 19 & M. Sas. & 1 y. $4 \mathrm{~m}$. & " & $2^{\prime \prime}$ & \\
\hline 20 & R. Hor. & $1 \mathrm{y} .7 \mathrm{~m}$. & Bronchopneumonia & $2^{\prime \prime}$ & \\
\hline 21 & K. Suz. & $1 \mathrm{y} .8 \mathrm{~m}$. & $"$ & $2^{\prime \prime}$ & \\
\hline 22 & K. Chi. & $2 y$. & $"$ & $4^{\prime \prime}$ & Since 1 month ill. \\
\hline 23 & M. End. & $9 \mathrm{y} .2 \mathrm{~m}$. & Asthma bronchiale & $2^{\prime \prime}$ & \\
\hline 24 & T. Ik. & $21 \mathrm{y}$ & Dysmenorihoe & $2^{\prime \prime}$ & \\
\hline 25 & Y. Um. & $10 \mathrm{y} .11 \mathrm{~m}$ & Nephritis acuta & $2 "$ & \\
\hline 26 & S. End. & 8 y. 2 m. & Chorea minor & $2^{\prime \prime}$ & \\
\hline 27 & K. Tom. & $3 y$ & Aphasia & $2^{\prime \prime}$ & \\
\hline 28 & S. Mat. & $2 y .5 \mathrm{~m}$. & Idiocy & $2^{\prime \prime}$ & \\
\hline 29 & S. Chi. & 9 y. $7 \mathrm{~m}$. & Epilepsia & $2^{\prime \prime}$ & \\
\hline 30 & S. Tak. & $1 \mathrm{y} .10 \mathrm{~m}$. & Hydrocephalus & $2^{\prime \prime}$ & \\
\hline 31 & S. Hin. & $50 y$. & Morbus Weilli & $3^{\prime \prime}$ & \\
\hline 32 & K. Ak. & 8 y. $9 \mathrm{~m}$. & Influenza & $3^{\prime \prime}$ & $\begin{array}{l}\text { High fever has conti- } \\
\text { nued long days with } \\
\text { no appetite. }\end{array}$ \\
\hline 33 & Z. Tak. & $9 \mathrm{y} .8 \mathrm{~m}$ & Typlus abdominalis & $2^{\prime \prime}$ & \\
\hline 34 & K. Sas. & $12 \mathrm{y} .1 \mathrm{~m}$. & " $\quad "$ & $2^{\prime \prime}$ & \\
\hline 35 & K. Tak. & $14 \mathrm{y}$. & $\begin{array}{l}\text { Typhus abdominalis }+ \\
\text { beriberi }\end{array}$ & $3^{\prime \prime}$ & \\
\hline 36 & M. Kud. & $7 \mathrm{y} .1 \mathrm{~m}$. & Morbilli & $2^{\prime \prime}$ & \\
\hline 37 & K. Tak. & $6 \mathrm{y} .11 \mathrm{~m}$. & " & $2^{\prime \prime}$ & \\
\hline 38 & A. Hox. & $1 \mathrm{y} .8 \mathrm{~m}$ & $"$ & $2^{\prime \prime}$ & \\
\hline 39 & S. Shi. & $13 \mathrm{y} .4 \mathrm{~m}$. & Scarlatina & $2^{\prime \prime}$ & \\
\hline 40 & S. Ob. & $1 \mathrm{y} .6 \mathrm{~m}$ & Erythema infectiosum & $2^{\prime \prime}$ & \\
\hline 41 & M. Sug. & $8 \mathrm{y}$. & Diphtheria & $2^{\prime \prime}$ & \\
\hline 42 & S. Kur. & $2 \mathrm{y} .11 \mathrm{~m}$. & $"$ & $2^{\prime \prime}$ & \\
\hline 43 & T. Kain. & $1 \mathrm{y} .7 \mathrm{~m}$. & Pleuritis tuberculosa & $2^{\prime \prime}$ & \\
\hline 44 & I. Shi. & $2 \mathrm{y} .1 \mathrm{~m}$ & , $\quad "$ & $2^{\prime \prime}$ & \\
\hline 45 & Y. Kum. & $5 \mathrm{y} .6 \mathrm{~m}$. & $", \quad \quad "$ & $\mathbf{9}^{\prime \prime}$ & \\
\hline 46 & Y. Noz. & $8 \mathrm{y} .1 \mathrm{~m}$. & Latent tuberculosis & $2^{\prime \prime}$ & \\
\hline
\end{tabular}




\begin{tabular}{|c|c|c|c|c|c|}
\hline No. & Name & $\begin{array}{c}\text { Age } \\
\mathrm{d} .=\text { days } \\
\mathrm{m} .=\text { months } \\
\mathrm{g}=\text { years }\end{array}$ & Diagnosis & $\begin{array}{l}\text { Stain- } \\
\text { time }\end{array}$ & Remark \\
\hline 47 & H. Fuj. & $11 y$. & Latent tuberculosis & $2^{\prime \prime}$ & \\
\hline 48 & Z. As. & $4 \mathrm{y} .1 \mathrm{~m}$ & Tuberculosis of abdomen & $2^{\prime \prime}$ & \\
\hline 49 & Y. Sui. & 6 y. $9 \mathrm{~m}$ & Pulmonary tuberculosis & $2^{\prime \prime}$ & \\
\hline 50 & K. Nag. & $5 \mathrm{y} .4 \mathrm{~m}$. & Latent tuberenlosis & $2 / \prime$ & \\
\hline 51 & M. Tan. & 12 y. $2 \mathrm{~m}$ & Spondylitis tuberculosa & $2^{\prime \prime}$ & \\
\hline 52 & Y. Suz. & $5 \mathrm{y} .2 \mathrm{~m}$ & Latent tuberculosis & $2 / \prime$ & \\
\hline $\mathbf{5 3}$ & $\mathbf{K} . \mathbf{A b}$ & $10 \mathrm{y} .10 \mathrm{~m}$ & $" \quad \quad "$ & $2^{\prime \prime}$ & \\
\hline 54 & T. Nuk. & $9 y$. & 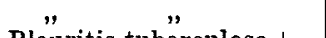 & $2^{\prime \prime}$ & \\
\hline 55 & Z. Mak. & 8 y. $2 \mathrm{~m}$. & $\begin{array}{l}\text { Pleuritis tuberculosa }+ \\
\text { pulmonary tuberculosis }\end{array}$ & $2^{\prime \prime}$ & \\
\hline 56 & H. Kan. & $8 \mathrm{y} .2 \mathrm{~m}$. & Latent tuberculosis & $2 \prime \prime$ & \\
\hline 57 & F. Gom. & $8 \mathrm{y} .4 \mathrm{~m}$. & $\begin{array}{l}\text { Pulmonary and abdomi- } \\
\text { nal tuberculosis }\end{array}$ & $2^{\prime \prime}$ & \\
\hline 58 & K. Tak. & $6 \mathrm{y}$. & Abdominal tuberculosis & $2^{\prime \prime}$ & \\
\hline 59 & T. Um. & $10 \mathrm{y}$. & Spondylitis tuberculosa & $2 "$ & \\
\hline 60 & S. Chi. & $1 \mathrm{y}$ & $\begin{array}{l}\text { Lues congenita }+ \\
\text { dispepsia }\end{array}$ & $2 \prime \prime$ & \\
\hline 61 & Y. Sek. & $2 \mathrm{y} .2 \mathrm{~m}$. & Lues congenita & $2^{\prime \prime}$ & \\
\hline 62 & T. Sat. & $9 \mathrm{~m}$ & $" \quad$ " & $2^{\prime \prime}$ & \\
\hline 63 & I. Iak. & $1 \mathrm{~g} .6 \mathrm{~m}$. & Dyspepsia acuta & $2 " \prime$ & \\
\hline 64 & A. Ko. & 2 y. $10 \mathrm{~m}$. & " " " & $2 " \prime$ & \\
\hline 65 & M. Saw. & $2 \mathrm{y} .2 \mathrm{~m}$ & $"$ & $2 " \prime$ & \\
\hline 66 & S. Nak. & $3 y$. & $"$ & $2 "$ & \\
\hline 67 & A. Nak. & $3 \mathrm{y} .11 \mathrm{~m}$. & $"$ & $2 \prime \prime$ & \\
\hline 68 & M. Kim. & $2 \mathrm{y} .2 \mathrm{~m}$ & $"$ & $2^{\prime \prime}$ & \\
\hline 69 & C. Ish. & $8 \mathrm{y} .3 \mathrm{~m}$. & Abscess in abdomen & $2^{\prime \prime}$ & \\
\hline 70 & M. Kat. & $12 \mathrm{y}$. & Healthy & $2^{\prime \prime}$ & \\
\hline 71 & M. Tak. & $21 \mathrm{y}$ & " & 2" & \\
\hline 72 & S. Shi. & $27 \mathrm{y}$. & " & $2 \prime \prime$ & \\
\hline 73 & S. Sat. & $8 \mathrm{y} .16 \mathrm{~m}$. & $"$ & $2 " \prime$ & \\
\hline 74 & T. Sat. & $28 \mathrm{y}$ & $"$ & $2^{\prime \prime}$ & \\
\hline 75 & H. Tan. & $20 \mathrm{y}$ & $"$ & $2 " \prime$ & \\
\hline 76 & Y. Kan. & $6 \mathrm{y} .1 \mathrm{~m}$ & Abdominal pain & $2 "$ & \\
\hline 77 & H. Sat. & $1 \mathrm{y} .2 \mathrm{~m}$. & $\begin{array}{l}\text { Tendency of cramp }+ \\
\text { obstipation }\end{array}$ & $2^{\prime \prime}$ & \\
\hline 78 & E. Tak. & $10 \mathrm{y}$. & Incontinentia albi & $2 \prime \prime$ & \\
\hline 79 & T. Tom. & $6 \mathrm{y} .9 \mathrm{~m}$ & Head-ache + anorexia & $2^{\prime \prime}$ & \\
\hline 80 & K. Has, & $2 \mathrm{y} .5 \mathrm{~m}$. & Idiopathic cramp & $2^{\prime \prime}$ & \\
\hline 81 & S. Fuj. & $5 \mathrm{y} .6 \mathrm{~m}$ & Myelogenic lenkemia & $2 / \prime$ & \\
\hline 82 & R. Suz. & $10 \mathrm{y} .3 \mathrm{~m}$. & Lymphatic leukemia & $2 \prime \prime$ & \\
\hline 83 & T. Sat. & $4 \mathrm{y} .2 \mathrm{~m}$ & Purpura & $2^{\prime \prime \prime}$ & \\
\hline 84 & T. Mom. & $10 \mathrm{y} .11 \mathrm{~m}$. & Hemorrhagic diathesis & $2^{\prime \prime}$ & \\
\hline 85 & T. Kiu. & $27 \mathrm{y}$ & Pregnancy & $2^{\prime \prime}$ & $\begin{array}{l}\text { A rakawa's reaction: } \\
\text { both sides utterly } \\
\text { negativ. }\end{array}$ \\
\hline 86 & S. Sat. & $11 \mathrm{y} .3 \mathrm{~m}$. & $\begin{array}{l}\text { White hair + nystagmus } \\
\text { +excited patellal reflex } \\
\text { + small stature. }\end{array}$ & $2 / \prime$ & \\
\hline 87 & S. Ui. & $11 \mathrm{~g} .4 \mathrm{~m}$. & " n $"$ & $2 \prime \prime$ & \\
\hline 88 & K. Ai. & $11 \mathrm{y}$. & Tumour of brain & $2 / \prime$ & \\
\hline 89 & K. Wak. & $8 \mathrm{y}$. & Doubt of tumour of brain & $2^{\prime \prime}$ & \\
\hline
\end{tabular}

(Table VII), and the "two seconds method" in the other series (Table VIII); so there can be only an indirect comparison, not a direct one. 
In the former series with the "three seconds method," there were 35 cases of different diseases, and three cases (Case No. 11, Case No. 20 and Case No. 23 in Table VII) among them that showed a prolongation, suffering evidently from avitaminosis $\mathbf{B}$.

As to healthy individuals, there were 31 cases tested with the "three seconds method." There were two cases with a prolongation (Cf. Table III). One mother was secreting milk utterly negative to the Arakawa's reaction and the other whose infant showed a prolongation (5 seconds) of the shortest peroxidase stain-time.

In the latter series with the "two seconds method," 83 cases of different diseases were put to the test. And there were 4 cases (Case No. 22, Case No. 31, Case No. 32 and Case No. 35 in Table VIII) that showed a prolongation of the shortest peroxidase stain-time; it was evident that all of them suffered from B-vitamin-deficit.

Again 84 apparently healthy individuals were tested with the "two seconds method," and there were 4 cases with a prolongation of the shortest peroxidase stain-time (Cf. Table VI), all of whom were secreting milk utterly negative to the Arak a wa's reaction.

It will thus be concluded from either of these two methods that a prolongation of the shortest peroxidase stain-time is a sign of avitaminosis B.

TABLE IX.

Relation between the Age and the Shortest Peroxidase Stain-time (Complyed from Tabs. I, II, III, IV, $V$ and VI of my own experience)

\begin{tabular}{|c|c|c|c|c|c|}
\hline \multirow{2}{*}{$\begin{array}{c}\text { Age } \\
\text { m. }=\text { months } \\
y==\text { years }\end{array}$} & \multirow{2}{*}{$\begin{array}{l}\text { Number of } \\
\text { tested cases }\end{array}$} & \multicolumn{2}{|c|}{ Prolonged } & \multicolumn{2}{|c|}{ Not prolonged } \\
\hline & & $\begin{array}{c}\text { Number of } \\
\text { cases }\end{array}$ & $\%$ & $\begin{array}{c}\text { Number of } \\
\text { cases }\end{array}$ & $\%$ \\
\hline $1 \mathrm{~m}$. & 12 & 9 & 75.0 & 3 & 25.0 \\
\hline $2 \mathrm{~m}$. & 41 & 25 & 60.9 & 16 & 39.1 \\
\hline $3 \mathrm{~m}$ & 25 & 9 & 36.0 & 16 & 64.0 \\
\hline $4 \mathrm{~m}$. & $y$ & 3 & 33.3 & 6 & 66.7 \\
\hline $5 \mathrm{~m}$. & 10 & 4 & 40.0 & 6 & 60.0 \\
\hline $6 \mathrm{~m}$. & 12 & 2 & 16.6 & 10 & 83.4 \\
\hline $7 \mathrm{~m}$ & 10 & 2 & 20.0 & 8 & 80.0 \\
\hline $8 \mathrm{~m}$ & 9 & 4 & 44.4 & 5 & 55.6 \\
\hline $9 \mathrm{~m}$ & 3 & 1 & 33.3 & 2 & 66.7 \\
\hline $10 \mathrm{~m}$ & 3 & 0 & 0 & 3 & 100.0 \\
\hline $11 \mathrm{~m}$. & 4 & 1 & 25.0 & 3 & 75.0 \\
\hline $12 \mathrm{n}$, & 10 & 4 & 40.0 & 6 & 60.0 \\
\hline $2 y$. & 23 & 7 & 30.4 & 16 & 69.6 \\
\hline $3 y$. & 1 & 0 & 0 & 1 & 100.0 \\
\hline Total & 172 & 71 & 41.2 & 101 & 58.8 \\
\hline
\end{tabular}


TABLE $X$.

Relation between the Age and the Shortest Peroxidase Stain-time (Complyed from Tabs. I and II of Chiba)

\begin{tabular}{|c|c|c|c|c|c|}
\hline \multirow{2}{*}{$\begin{array}{c}\text { Age } \\
\mathrm{m}_{.}=\text {months } \\
\mathrm{y} .=\text { years }\end{array}$} & \multirow{2}{*}{$\begin{array}{l}\text { Number of } \\
\text { tested cases }\end{array}$} & \multicolumn{2}{|c|}{ Prolonged } & \multicolumn{2}{|c|}{ Not prolonged } \\
\hline & & $\begin{array}{c}\text { Number of } \\
\text { cases }\end{array}$ & $\%$ & $\begin{array}{c}\text { Number of } \\
\text { cases }\end{array}$ & $\%$ \\
\hline $1 \mathrm{~m}$. & 34 & 14 & 41.1 & 20 & 58.9 \\
\hline $2 \mathrm{~m}$. & 23 & 9 & 39.1 & 14 & 60.9 \\
\hline $3 \mathrm{~m}$. & 13 & 2 & 15.3 & 11 & 84.7 \\
\hline $4 \mathrm{~m}$ & 9 & 3 & 33.3 & 6 & 66.7 \\
\hline $5 \mathrm{~m}$. & 5 & 1 & 20.0 & 4 & 80.0 \\
\hline $6 \mathrm{~m}$ & 14 & 7 & 50.0 & 7 & 50.0 \\
\hline $7 \mathrm{~m}$. & 5 & 1 & 20.0 & 4 & 80.0 \\
\hline $8 \mathrm{~m}$. & 3 & 0 & 0 & 3 & 100.0 \\
\hline $9 \mathrm{~m}$. & 2 & 2 & 100.0 & 0 & 0 \\
\hline $10 \mathrm{~m}$. & 6 & $\overline{3}$ & 50.0 & 3 & 50.0 \\
\hline $11 \mathrm{~m}$. & 6 & 4 & 66.6 & 2 & 33.4 \\
\hline $12 \mathrm{~m}$. & 3 & 2 & 66.6 & 1 & 33.4 \\
\hline $2 y$ & 46 & 15 & 32.6 & 31 & 67.4 \\
\hline $3 y$. & 1 & 0 & 0 & 1 & 100.0 \\
\hline Total & 170 & 63 & 37.0 & 107 & 63.0 \\
\hline
\end{tabular}

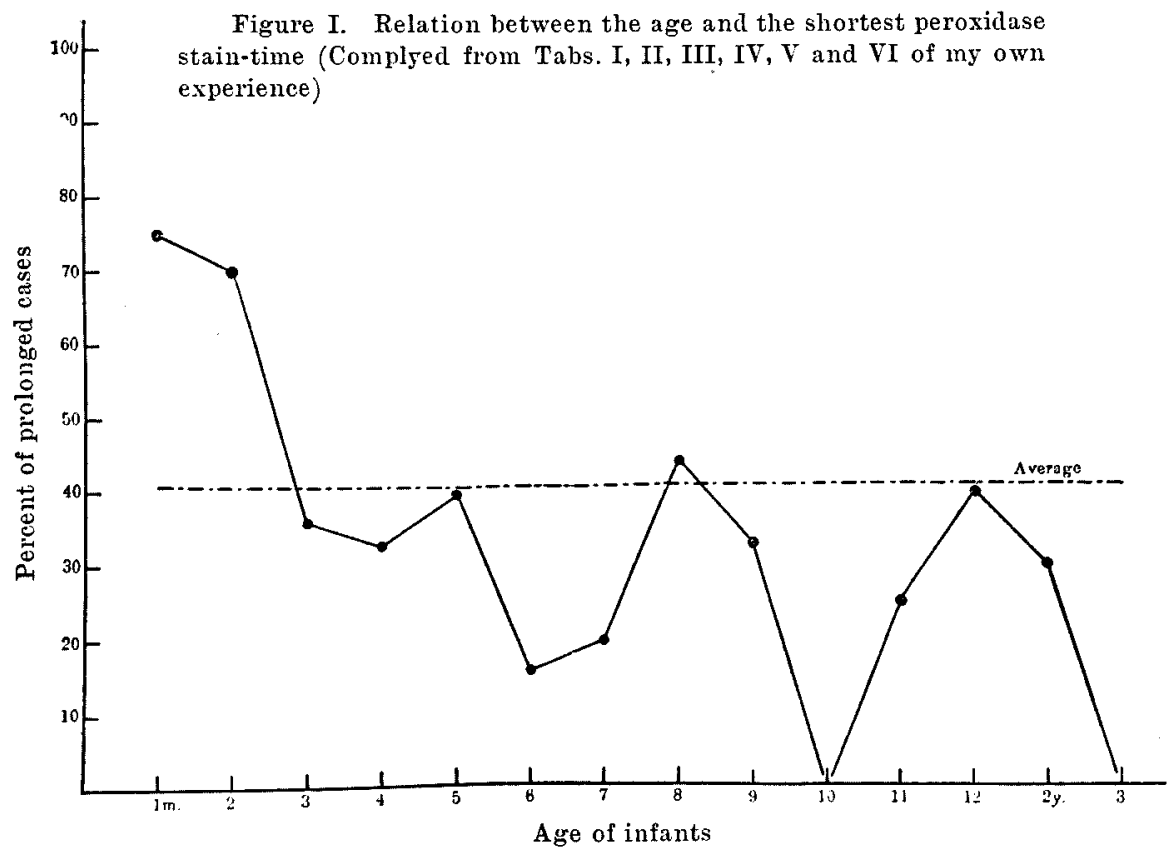


Figure II. Relation between the age and the shortest peroxidase stain-time (Complyed from Tabs. I and II of Chiba's experience)

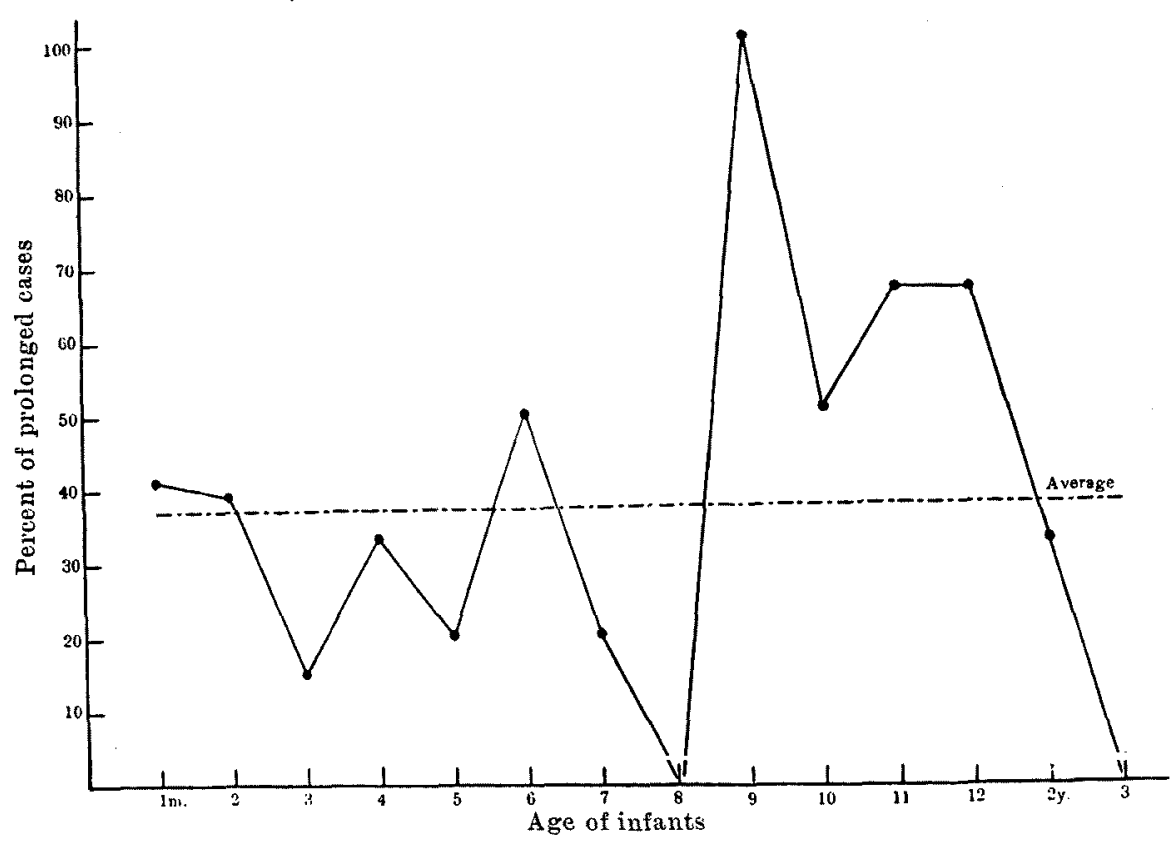

We have seen from the paper of Chiba and $\mathrm{Ab} \mathrm{e}^{5}$ that mothers with a deficit in vitamin $B$ can make their sucklings more $B$-avitaminotic than their own bodies. I have, as already stated, experienced the same thing in my investigation. This fact may be explained from a difference in age. If this way of explanation is applied to infants themselves, there must be some difference concerning the prolongation of the shortest peroxidase stain-time according to the difference of age, namely, according as infants are older or younger. As will be seen from Tab. IX and Fig. I, younger infants are surely more disposed to the prolongation than older ones, though the relation is not so clearly seen from Tab. X and Fig. II, as from Tab. IX and Fig. I. At any rate, it seems that older people are less prone to the alleged prolongation than infants are.

\section{Conclusions.}

1. The shortest stain-time for copper peroxidase reaction (of neutrophile leucocytes) has been considered by K. Suzuki to be nor- 
mal in human cases, if it is three seconds. But my experience shows that it is two seconds instead of three. In other words, neutrophiles of human blood will begin to take peroxidase stain just perceptibly in two seconds.

2. By this method of short-timed peroxidase reaction a similar conclusion as has been made by $\mathrm{Chiba}$ and $\mathrm{Abe}$ is attained. In individuals with B-vitamin-deficit or with diseases related to it, a prolongation of the short stain-time will occur; the longest prolongation $I$ have ever experienced is 8 seconds (in contrast to 2 seconds as normal). The prolongation will be made good if vitamin B is administered in a fair amount.

3. The prolongation of the shortest peroxidase stain-time occurs more remarkably in younger individuals. This explains the fact that it is found much more frequent in suckling infants than in their mothers, more frequent in younger infants than in older ones. 